\title{
Natural history of human aberrant crypt foci and correlation with risk factors for colorectal cancer
}

\author{
HIDENORI OHKUBO $^{1}$, HIROKAZU TAKAHASHI ${ }^{1}$, EIJI YAMADA ${ }^{1}$, EIJI SAKAI ${ }^{1}$, \\ TAKUMA HIGURASHI ${ }^{1}$, TAKASHI UCHIYAMA ${ }^{1}$, KUNIHIRO HOSONO $^{1}$, HIROKI ENDO $^{1}$, \\ MASATAKA TAGURI $^{2}$ and ATSUSHI NAKAJIMA ${ }^{1}$ \\ ${ }^{1}$ Gastroenterology Division and ${ }^{2}$ Biostatistics and Epidemiology, Yokohama City \\ University School of Medicine, Yokohama 236-0004, Japan
}

Received November 25, 2011; Accepted December 30, 2011

DOI: $10.3892 / o r .2012 .1631$

\begin{abstract}
Although aberrant crypt foci (ACF) are estimated to have potential usefulness as a biomarker for colorectal carcinoma (CRC), this remains uncertain because the natural history of ACF has not been well-clarified. To determine the usefulness of ACF as a surrogate marker for CRC, it is necessary to understand the natural history of ACF. A total of 82 subjects who underwent total colonoscopy and whose ACF number was examined at least 2 times at Yokohama City University Hospital were enrolled. We retrospectively evaluated the changes in the ACF number at four different surveillance periods ( 6 months, 1 year, 2 years, 3 years) and in groups with and without colorectal neoplasms. Furthermore, we classified the subjects into an increased ACF group and a no change/decreased ACF group, and investigated the relationship between the changes in the ACF number and known risk factors for CRC. No significant differences were observed in the ACF number between the first and second observations in any surveillance period groups, and in the groups classified according to the presence or absence of colorectal neoplasms. There were no significant differences between the increased and no change/decreased ACF group in terms of gender, smoking habit, current alcohol consumption, age, BMI, HbAlc or serum triglyceride level (TG), whereas a significant difference between the groups was observed in the serum total cholesterol level (TC) $(\mathrm{P}=0.012)$. ACF are a reliable surrogate
\end{abstract}

Correspondence to: Dr Atsushi Nakajima, Gastroenterology Division, Yokohama City University School of Medicine, 3-9 Fuku-ura, Kanazawa-ku, Yokohama 236-0004, Japan

E-mail: nakajima-tky@umin.ac.jp

Abbreviations: ACF, aberrant crypt foci; CRC, colorectal carcinoma; HMCC, high-magnification chromoendoscopy; TC, serum total cholesterol level; TG, serum triglyceride level; MS, metabolic syndrome

Key words: aberrant crypt foci, natural history, colorectal neoplasms, risk factor marker that are not affected by any risk factors for adenomas or CRC, except TC, and may therefore be considered as a useful marker in chemopreventive trials.

\section{Introduction}

Aberrant crypt foci (ACF), which are clusters of colorectal crypts with abnormal morphology, were initially identified as the earliest recognizable lesions on the colonic mucosa of rodents exposed to colorectal carcinogens (1-3). Numerous animal studies have demonstrated ACF as an important predictor of colorectal carcinoma (CRC) development. In humans, ACF were first identified in the colonic mucosa of surgical specimens, shortly after their description in animals. More recently, they were identified in vivo by high-magnification chromoendoscopy (HMCC). ACF have emerged over the last decade as putative precursors to colorectal adenomas, and therefore as a potential biomarker for CRC in humans.

Cross-sectional studies have shown a higher rate of ACF occurrence in patients with $\mathrm{CRC}$ and adenomas as compared with that in normal patients, which suggests the potential usefulness of ACF as a surrogate marker of CRC (4). However, there are many questions raised by recent studies, such as whether ACF progress spontaneously or not, and whether ACF growth might be correlated with known risk factors for CRC $(1,5)$. There is only one literature report relating to the natural history of ACF, by Schoen et al in 2008 (2). This study examined the reproducibility of ACF detection over a 1-year period of observation, however, only $43 \%$ of ACF could be specifically re-identified, and more than $50 \%$ of the subjects had new ACF. The considerable dynamic to ACF detection in this study may be due to progression or regression of the $\mathrm{ACF}$ themselves, lack of adequate experience of the examiners in performing the examination for ACF, and inaccuracy in identifying the size and location of the ACF (2). Schoen et al also showed difficulty in re-identifying ACF at the 1-year examination (2). As a result, the natural history of ACF has not yet been well-clarified. Knowledge of the natural history of ACF is needed to understand their potential usefulness as a surrogate marker for CRC.

Furthermore, previous studies have shown the existence of a relationship between the risk factors for CRC and the size 
and number of ACF (4,6-9), however, there are no studies showing the relationship between the known risk factors for $\mathrm{CRC}$ and the changes in the number of ACF over time. Very little is known about the factors that promote or inhibit ACF growth in humans.

In this study, we retrospectively examined the changes in the number of ACF over time and investigated their correlation with known risk factors for CRC in 82 subjects.

\section{Materials and methods}

Study population. Patients who underwent a total colonoscopy and examination of the ACF number at least 2 times at Yokohama City University Hospital from 2004 to 2010 were eligible to participate in this study. The following were the patient exclusion criteria; history of familial adenomatous polyposis, hereditary non-polyposis colorectal cancer, inflammatory bowel disease, invasive cancer, prior large-bowel resection except appendectomy, and regular aspirin or NSAID use. A total of 82 subjects were enrolled in this study. The first and the second examinations were performed by the same examiner after the different four surveillance periods (6 months, 1 year, 2 years, 3 years).

Data sampling. We collected data including the age, gender, comorbidities, past medical history, body mass index (BMI), daily alcohol consumption, and smoking habit from the past records. Subjects were also reviewed for information on the blood data, including HbAlc, serum total cholesterol levels (TC), and serum triglyceride levels (TG), which were calculated just before the first examination.

Endoscopy. All subjects were prepared for endoscopy with a polyethylene glycol-based solution. Total colonoscopy was performed before the examination for ACF. Data on the histology of the polyps removed or biopsied at colonoscopy or previously were obtained from the pathology records. A Fujinon EC-490ZW5/M colonoscope (Fujinon Toshiba ES System Co., Ltd., Tokyo, Japan) was used to conduct magnifying chromoendoscopy. Because of the difficulty in assessment of the number and size of ACF of the entire colon, we examined $\mathrm{ACF}$ only from the anal verge to the middle Houston valve. Methylene blue dye $(0.2 \%)$ was administered to coat the mucosal surface, followed by adequate washing with warm tap water. After 2 min of staining the mucosa, the excess dye was removed carefully by washing with water, and then observation for ACF was commenced. We examined the size and number of the ACF, and investigated the changes in the number of ACF between the first and second observations.

Criteria used to identify and define ACF on endoscopy. ACF are clusters of crypts visualized after staining with methylene blue that appear 'different' from the surrounding mucosa. However, what characterizes them as different varies across the literature. Most commonly, ACF are identified as clusters of crypts that stain darker than the surrounding normal mucosa $(4,7,11,18)$. However, larger crypt size $(4,7)$, raised appearance $(7,12,18)$, thicker epithelial lining (4), dilated or slit-like crypt lumen $(12,19)$, and increased pericryptal area (19) as compared

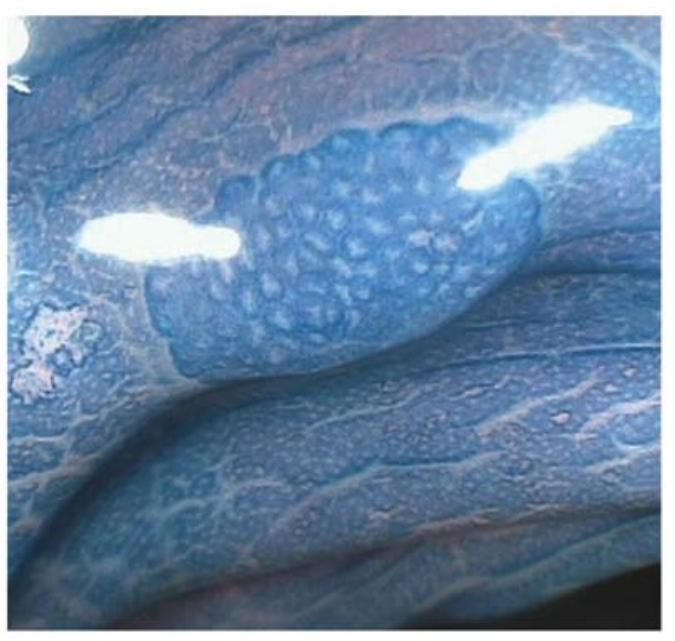

Figure 1. Endoscopic photograph of an ACF with the typical features; clusters of crypts that stain darker than the surrounding normal mucosa, larger crypt size, raised appearance, thicker epithelial lining, dilated or slit-like crypt lumen, and increased pericryptal area.

Table I. Number and mean age (years) of the subjects enrolled in this study.

\begin{tabular}{lcccc}
\hline & Total & Male & Female & P-value $^{\text {a }}$ \\
\hline $\begin{array}{l}\text { No. of subjects } \\
\text { Mean age } \pm \text { SD }\end{array}$ & 62 & 53 & 29 & \\
\hline
\end{tabular}

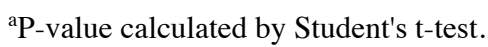

with the surrounding normal mucosa are other frequently used criteria to identify ACF (5). We also used these characteristics as criteria for the diagnosis of ACF. An endoscopic photograph of an ACF with the typical features is shown in Fig. 1.

Statistical analysis. First, we used a paired t-test to evaluate the changes in the number of ACF in each surveillance period. At the same time, we assessed whether or not the present or past colorectal neoplasms were correlated with the change in the number of ACF.

Next, we classified the patients into 2 groups (increased ACF group and no change/decreased ACF group), and examined whether there were significant differences between these two groups in the known risk factors for CRC, such as gender, smoking habit and current alcohol consumption by using the $\chi^{2}$ test, and the mean age, BMI, HbAlc, TC and TG by using the Student's t-test.

\section{Results}

Changes in ACF number in the four different surveillance periods and in the groups with and without colorectal neoplasms. A total of 82 subjects, 53 men and 29 women, aged 35 to 81 years, were enrolled in this study, as shown in Table I. At the first examination, the mean $\pm \mathrm{SD}$ age of the participants was $63.6 \pm 11.1$ years (male, $63.6 \pm 10.4$, female, $63.5 \pm 12.6$, $\mathrm{P}=0.83$ ). 
Table II. Changes in the numbers of aberrant crypti foci (ACF) in the four different surveillance periods and in the groups with and without colorectal neoplasms (mean $\pm \mathrm{SD})$.

\begin{tabular}{|c|c|c|c|c|}
\hline & \multirow[b]{2}{*}{$\mathrm{N}$} & \multicolumn{2}{|c|}{ No. of ACF } & \multirow[b]{2}{*}{ P-value } \\
\hline & & First observation & Second observation & \\
\hline \multicolumn{5}{|c|}{ Classification according to the four different surveillance periods } \\
\hline 6 months & 31 & $5.13 \pm 5.87$ & $4.81 \pm 5.04$ & 0.64 \\
\hline 1 year & 30 & $3.87 \pm 4.61$ & $3.90 \pm 4.47$ & 0.96 \\
\hline 2 years & 15 & $4.67 \pm 4.12$ & $3.93 \pm 4.40$ & 0.15 \\
\hline 3 years & 6 & $4.50 \pm 4.05$ & $4.00 \pm 3.85$ & 0.72 \\
\hline Total & 82 & $4.53 \pm 4.97$ & $4.26 \pm 4.58$ & 0.46 \\
\hline \multicolumn{5}{|c|}{ Classification according to with and without colorectal neoplasms } \\
\hline Normal group & 36 & $3.68 \pm 3.11^{\mathrm{b}}$ & $3.26 \pm 3.54$ & 0.23 \\
\hline Adenoma group & 36 & $3.89 \pm 4.03^{\mathrm{b}}$ & $4.42 \pm 4.32$ & 0.39 \\
\hline Carcinoma group & 10 & $7.30 \pm 7.48^{\mathrm{b}}$ & $6.00 \pm 7.19$ & 0.35 \\
\hline Total & 82 & $4.53 \pm 4.97$ & $4.26 \pm 4.58$ & 0.46 \\
\hline
\end{tabular}

Table III. Observation period, age and gender of the 3 groups classified according to with and without colorectal neoplasms $($ mean $\pm \mathrm{SD})$.

\begin{tabular}{lcccc}
\hline & Total & Normal & Adenoma & Carcinoma \\
\hline Observation period (years) $^{\mathrm{a}}$ & $1.1 \pm 0.6$ & $1.4 \pm 0.9$ & $1.0 \pm 0.6$ & $0.8 \pm 0.2$ \\
Age (years) $^{\mathrm{b}}$ & $63.6 \pm 11.1$ & $63.8 \pm 12.7$ & $65.0 \pm 9.7$ & $58.9 \pm 8.3$ \\
Gender (Male/female) & $82(53 / 29)$ & $36(23 / 13)$ & $36(24 / 12)$ & $10(6 / 4)$ \\
\hline
\end{tabular}

${ }^{\mathrm{a}} \mathrm{P}=0.06$ and ${ }^{\mathrm{b}} \mathrm{P}=0.29$, calculated by Kruskal-Wallis test.

Table II summarizes the changes in the number of ACF in the four different surveillance periods and in the groups with and without colorectal neoplasms. The numbers of ACF at the first and second observations were 5.13 $\pm 5.87 / 4.81 \pm 5.04$ $(\mathrm{P}=0.64)$ in the 6 months group, 3.87 $\pm 4.61 / 3.90 \pm 4.47(\mathrm{P}=0.96)$ in the 1-year group, $4.67 \pm 4.12 / 3.93 \pm 4.40(\mathrm{P}=0.15)$ in the 2 -years group, and 4.50 $\pm 4.05 / 4.00 \pm 3.85(\mathrm{P}=0.72)$ in the 3 -years group. In total, there was an average of $4.53 \pm 4.97 / 4.26 \pm 4.58(\mathrm{P}=0.46)$ $\mathrm{ACF}$ present at the first and second observations. There were no significant differences between the first and second observations in each group or overall, as mentioned above, but this grouping did not take into account the potential variation related to the presence of colonic neoplasms. Therefore, we classified the patients into three groups according to whether or not they had present or past colorectal neoplasms (normal group, adenoma group and carcinoma group), and evaluated the changes in the number of ACF in each group. There was a mean of $3.68 \pm 3.11 / 3.26 \pm 3.54(\mathrm{P}=0.23) \mathrm{ACF}$ observed at the first and second observations in the normal group, $3.89 \pm 4.03 / 4.42 \pm 4.32(\mathrm{P}=0.39)$ in the adenoma group, and $7.30 \pm 7.48 / 6.00 \pm 7.19(\mathrm{P}=0.35)$ in the carcinoma group. Thus, no significant changes in the number of ACF between the first and second observations were observed in any group, even the groups classified according to the presence or absence of colorectal neoplasms. On the other hand, there were significantly increased ACF observed at the first observation in the carcinoma group compared with those in the normal group, and those in the adenoma group $(\mathrm{P}=0.02, \mathrm{P}=0.03$ respectively). Although there were no statistically significant differences, more ACF were observed at the second observation in the carcinoma group than in the normal group and adenoma group.

Table III summarizes the mean observation period, age and gender of the 3 groups classified according to with and without colorectal neoplasms. The mean observation periods were $1.4 \pm 0.9$ years in the normal group, $1.0 \pm 0.6$ years in the adenoma group, and $0.8 \pm 0.2$ years in the carcinoma group, in which no statistically significant differences were observed $(\mathrm{P}=0.06)$. In addition, there were also no statistically significant differences observed in the mean age of these 3 groups $(\mathrm{P}=0.29)$.

Relationship between the changes in the number of ACF and known risk factors for $C R C$. Finally, we analyzed whether or not each known risk factor for CRC was correlated with 
Table IV. Relationship between the changes in the number of ACF number and known risk factors for CRC.

\begin{tabular}{|c|c|c|c|c|}
\hline & \multicolumn{3}{|c|}{ No. of ACF } & \multirow[b]{2}{*}{ P-value } \\
\hline & Total & Increase & No change/decrease & \\
\hline No. of subjects & 82 & 28 & 54 & \\
\hline Gender (Male/female) & $53 / 29$ & $15 / 13$ & $38 / 16$ & $0.13^{\mathrm{a}}$ \\
\hline $\begin{array}{l}\text { Smoking, n (\%) } \\
\text { Yes } \\
\text { No }\end{array}$ & $\begin{array}{l}37(45) \\
45(55)\end{array}$ & $\begin{array}{l}11(39) \\
17(61)\end{array}$ & $\begin{array}{l}26(48) \\
28(52)\end{array}$ & $0.44^{\mathrm{a}}$ \\
\hline $\begin{array}{l}\text { Alcohol intake, n (\%) } \\
\text { Yes } \\
\text { No }\end{array}$ & $\begin{array}{l}43(52) \\
39(48)\end{array}$ & $\begin{array}{l}11(39) \\
17(61)\end{array}$ & $\begin{array}{l}32(59) \\
22(41)\end{array}$ & $0.09^{\mathrm{a}}$ \\
\hline Mean age \pm SD (years) & $63.6 \pm 11.1$ & $61.6 \pm 9.2$ & $65.6 \pm 10.2$ & $0.09^{\mathrm{b}}$ \\
\hline $\mathrm{BMI} \pm \mathrm{SD}\left(\mathrm{kg} / \mathrm{m}^{2}\right)$ & $22.9 \pm 3.1$ & $22.9 \pm 2.7$ & $22.9 \pm 3.2$ & $0.97^{\mathrm{b}}$ \\
\hline $\mathrm{HbA} 1 \mathrm{c} \pm \mathrm{SD}(\%)$ & $5.66 \pm 1.03$ & $5.63 \pm 0.95$ & $5.67 \pm 1.07$ & $0.89^{\mathrm{b}}$ \\
\hline $\mathrm{TC} \pm \mathrm{SD}(\mathrm{mg} / \mathrm{dl})$ & $211.6 \pm 34.1$ & $225.5 \pm 34.4$ & $204.5 \pm 32.7$ & $0.012^{\mathrm{b}, \mathrm{c}}$ \\
\hline $\mathrm{TG} \pm \mathrm{SD}(\mathrm{mg} / \mathrm{dl})$ & $146.8 \pm 71.1$ & $162.0 \pm 90.7$ & $138.9 \pm 57.9$ & $0.19^{\mathrm{b}}$ \\
\hline
\end{tabular}

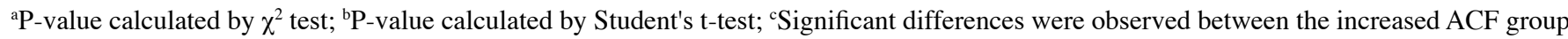
and the no change/decreased ACF in TC. BMI, body mass index; HbA1c, hemoglobin A1c; TC, serum total cholesterol; TG, serum triglyceride level.

the changes in the number of ACF (shown in Table IV). We divided the subjects into the increased ACF group and the no change/decreased ACF group so as to clarify what is associated with ACF increase. Subjects in whom the number of ACF had increased at least by one between the first and second observations were classified as the increased ACF group, and those in whom the number of ACF had decreased or not changed between the observations were classified as no change/decreased ACF group. There were 28 subjects in the increased ACF group, and 54 subjects in the no change/ decreased ACF group. The proportion of the subjects who smoked habitually was $39 \%$ in the former group, and $48 \%$ in the latter group. Similarly, the proportion of those who were current alcohol consumers was 39\% in the former group and $59 \%$ in the latter group. No significant differences were seen between the two groups in terms of gender, smoking habit, or daily alcohol intake. The other factors were as follows: mean age ; $61.6 \pm 9.2$ years old vs. $65.6 \pm 10.2$ years old, BMI; $22.9 \pm 2.7$ $\mathrm{kg} / \mathrm{m}^{2}$ vs. $22.9 \pm 3.2 \mathrm{~kg} / \mathrm{m}^{2}, \mathrm{HbAlc} ; 5.63 \pm 0.95 \%$ vs. $5.67 \pm 1.07 \%$, TC; $225.5 \pm 34.4 \mathrm{mg} / \mathrm{dl}$ vs. $204.5 \pm 32.7 \mathrm{mg} / \mathrm{dl}$, TG; $162.0 \pm 90.7$ $\mathrm{mg} / \mathrm{dl}$ vs. $138.9 \pm 57.9 \mathrm{mg} / \mathrm{dl}$. There were also no significant differences between the groups in the mean age, BMI, HbA1c or $\mathrm{TG}$, except $\mathrm{TC}(\mathrm{P}=0.012)$. In summary, there were no significant differences between the increased $\mathrm{ACF}$ group and the no change/decreased ACF in terms of gender, smoking habit, daily alcohol intake, age, BMI, HbA1c or TG, whereas a significant difference in the TC was observed between the two groups.

\section{Discussion}

CRC is a major neoplasm worldwide, and its prevalence and mortality have been increasing $(15,16)$. In particular, CRC is one of the most common causes of cancer-related death in developed countries (17). A paradigm shift from surveillance for early detection of cancer or adenomas to new preventive strategies, including chemoprevention, is needed to lower the burden of this disease (14). Several studies using HMCC for rectal ACF detection have reported a stepwise increase in the prevalence and frequency of rectal ACF in patients with a normal colon, adenoma and carcinoma (5-7,10-13). Because of the epidemiologic and genetic association of ACF with colorectal neoplasms, ACF may be a potential biomarker for CRC (2). For example, ACF could be used as endpoints in studies comparing individuals with and without risk factors for CRC and in studies evaluating chemopreventive agents. Because of the high prevalence of the ACF, studies using $\mathrm{ACF}$ as an endpoint can have a smaller sample size, and the dose-response relationship could be potentially assessed using the effect on the number of ACF (1). Recently, there have been several chemoprevention trials using ACF as a surrogate marker for CRC. Metformin has been reported to be an antidiabetes drug with a chemopreventive effect against CRC, not only in rodents but also in humans, by using ACF as a surrogate marker for CRC $(14,21)$.

If the natural variation of ACF is substantial, a chemoprevention agent must produce significantly greater regression of ACF than that which could be explained as a natural variation (2). However, there is only one previous report that has shown the natural changes in the number of ACF over time. Further knowledge of the natural history of ACF is necessary to understand their potential usefulness as a surrogate marker for CRC.

Our investigation of the natural variability of ACF in the 82 subjects showed that the number of ACF did not change significantly over time. When the subjects were divided 
according to four different surveillance periods from 6 months to 3 years, there were no significant differences between the first and second observation in any group. However, classification only according to the surveillance period is not sufficient to evaluate the natural variations of ACF, because having past or synchronous colorectal neoplasms itself may influence the fluctuation in the number of ACF over time. Takayama et al reported that both the number and percent of dysplastic ACF increased as one progressed from individuals with normal colons to those with adenomatous polyps and to those with CRC (4). Moreover, another report showed that persons with adenomas have a somewhat larger number of rectal ACF than those without, and that older age is a risk factor for ACF (7). From epidemiological studies so far, it has been established beyond doubt that persons with adenomas or carcinomas are likely to have more rectal ACF than those without. In order to rule out the variations of ACF which may be caused by the presence of colorectal neoplasms, we classified all the subjects into three groups according to whether or not they had present or past colorectal neoplasms (normal group, adenoma group, carcinoma group). Significantly increased ACF were observed at the baseline examination in the carcinoma group compared with those in the normal group, and those in the adenoma group, which is consistent with previous studies. On the other hand, no significant changes in the number of $\mathrm{ACF}$ over time were observed regardless of the presence or absence of colorectal neoplasms. A much longer observation period may lead to significant changes in the number of ACF in the carcinoma group, however in this study, no significant changes in the number of ACF were observed in each group at least during the observation period from 6 months to 3 years (mean, 1.1 years).

On the other hand, the factors that promote or inhibit ACF growth remain largely unknown in humans (7). However, it is predictable that some factors associated with the risk of colorectal adenomas and CRC may also affect the ACF growth because patients with colorectal neoplasms tend to have more ACF than those without. The metabolic syndrome (MS), which is an important risk factor for cardiovascular disease and contributes to morbidity and mortality through its main components, including diabetes mellitus, obesity, dyslipidemia and hypertension, has been linked to some malignancies, including CRC. MS is associated with an increased risk of colorectal adenoma, and this risk increases with the number of MS components (20). In addition, Takahashi et al demonstrated that obesity, particularly visceral fat accumulation, aging and a low plasma adiponectin level could correlate with colorectal carcinogenesis (22). In our study, we divided the subjects into the increased ACF group and the no change/decreased ACF group, and investigated the correlation between the changes in the number of $\mathrm{ACF}$ and known risk factors for $\mathrm{CRC}$, in order to elucidate the factors associated with ACF increase. As a result, TC was significantly increased in the increased $\mathrm{ACF}$ group as compared with that in the no change/decreased $\mathrm{ACF}$ group $(\mathrm{P}=0.012)$. Our results showed that an MS component, but not all components, was associated with ACF increase. In other words, the factors that promoted ACF growth were correlated with the risk factors for colorectal adenomas. In addition, our results also suggest that elevated TC is possible to increase the number of ACF in a relatively short period of
1.1 years average. Although the biological significance of ACF remains controversial, it is certain from previous studies that more ACF are present in patients with colorectal neoplasms than in those without $(4,7,23)$. Our results suggest that the prevention of hypercholesterolemia has the potential to reduce ACF formation, and may lead to reduction of colorectal neoplasms.

In conclusion, the novelty of our study lies in the findings that the number of ACF does not change significantly for at least 3 years (mean 1.1 years) regardless of the presence or absence of colorectal neoplasms, and that TC is the only factor associated with increment in the number of ACF over time. This suggests that ACF might be a reliable surrogate marker for CRC that is not affected by any risk factors for adenomas or CRC except TC, and could therefore be a useful marker in chemopreventive trials. At the same time, the prevention of hypercholesterolemia has the potential to reduce ACF formation and may lead to reduction of colorectal neoplasms.

\section{Acknowledgements}

The authors thank the nursing staff of the Yokohama City University Hospital for their assistance with the endoscopic procedures, and the Fujinon Corporation for providing the endoscopic equipment needed for the HMCC. This study was supported in part by a Grant-in-Aid for research on the ThirdTerm Comprehensive Control Research for Cancer from the Ministry of Health, Labour and Welfare, Japan, to A.N.

\section{References}

1. Gupta AK, Pretlow TP and Schoen RE: Aberrant crypt foci: what we know and what we need to know. Clin Gastroenterol Hepatol 5: 526-533, 2007

2. Schoen RE, Mutch M, Rall C, Dry SM, Seligson D, Umar A and Pinsky P: The natural history of aberrant crypt foci. Gastrointest Endosc 67: 1097-1102, 2008

3. Gupta AK, Pinsky P, Rall C, Mutch M, Dry S, Seligson D and Schoen RE: Reliability and accuracy of the endoscopic appearance in the identification of aberrant crypt foci. Gastrointest Endosc 70: 322-330, 2009.

4. Takayama T, Katsuki S, Takahashi Y, et al: Aberrant crypt foci of the colon as precursors of adenoma and cancer. N Engl J Med 339: 1277-1284, 1998.

5. Gupta AK and Schoen R: Aberrant crypt foci: are they intermediate endpoints of colon carcinogenesis in humans? Curr Opin Gastroenterol 25: 59-65, 2009.

6. Moxon D, Raza M, Kenney R, Ewing R, Arozullah A, Mason JB and Carroll RE: Relationship of aging and tobacco use with the development of aberrant crypt foci in a predominantly AfricanAmerican population. Clin Gastroenterol Hepatol 3: 271-278, 2005.

7. Rudolph RE, Dominitz JA, Lampe JW, et al: Risk factors for colorectal cancer in relation to number and size of aberrant crypt foci in humans. Cancer Epidemiol Biomarkers Prev 14: 605-608, 2005.

8. Stevens RG, Swede H, Heinen CD, et al: Aberrant crypt foci in patients with a positive family history of sporadic colorectal cancer. Cancer Lett 248: 262-268, 2007.

9. Stevens RG, Swede H and Daniel WR: Epidemiology of colonic aberrant crypt foci: review and analysis of existing studies. Cancer Lett 252: 171-183, 2007.

10. Seike K, Koda K, Oda K, et al: Assessment of rectal aberrant crypt foci by standard chromoscopy and its predictive value for colonic advanced neoplasms. Am J Gastroenterol 101: 1362-1369, 2006.

11. Hurlstone DP, Karajeh M, Sanders DS, Drew SK and Cross SS: Rectal aberrant crypt foci identified using high-magnificationchromoscopic colonoscopy: biomarkers for flat and depressed neoplasia. Am J Gastroenterol 100: 1283-1289, 2005. 
12. Adler DG, Gostout CJ, Sorbi D, Burgart LJ, Wang L and Harmsen WS: Endoscopic identification and quantification of aberrant crypt foci in the human colon. Gastrointest Endosc 56: $657-662,2002$.

13. Kukitsu T, Takayama T, Miyanishi K, et al: Aberrant crypt foci as precursors of the dysplasia-carcinoma sequence in patients with ulcerative colitis. Clin Cancer Res 14: 48-54, 2008.

14. Hosono K, Endo H, Takahashi H, et al: Metformin suppresses colorectal aberrant crypt foci in a short-term clinical trial. Cancer Prev Res 3: 1077-1083, 2010.

15. Jemal A, Siegel R, Xu J and Ward E: Cancer statistics, 2010. CA Cancer J Clin 60: 277-300, 2010.

16. Anderson WF, Umar A and Brawley OW: Colorectal carcinoma in black and white race. Cancer Metastasis Rev 22: 67-82, 2003.

17. Rougier P and Mitry E: Epidemiology, treatment and chemoprevention in colorectal cancer. Ann Oncol 14 (Suppl 2): ii3-ii5, 2003.

18. Yokota T, Sugano K, Kondo H, et al: Detection of aberrant crypt foci by magnifying colonoscopy. Gastrointest Endosc 46: 61-65, 1997.

19. Kim J, Ng J, Arozulllah A, Ewing R, Llor X, Carroll RE and Benya RV: Aberrant crypt focus size predicts distal polyp histopathology. Cancer Epidemiol Biomarkers Prev 17: 1155-1162, 2008.
20. Hu NC, Chen JD, Lin YM, Chang JY and Chen YH: Stepwise relationship between components of metabolic syndrome and risk of colorectal adenoma in a Taiwanese population receiving screening colonoscopy. J Formos Med Assoc 110: 100-108, 2011.

21. Hosono K, Endo H, Takahashi H, et al: Metformin suppresses azoxymethane-induced colorectal aberrant crypt foci by activating AMP-activated protein kinase. Mol Carcinog 49: 662-671, 2010.

22. Takahashi H, Takayama T, Yoneda $\mathrm{K}$, et al: Association of visceral fat accumulation and plasma adiponectin with rectal dysplastic aberrant crypt foci in a clinical population. Cancer Sci 100: 29-32, 2009.

23. Sakai E, Takahashi H, Kato S, et al: Investigation of the prevalence and number of aberrant crypt foci associated with human colorectal neoplasm. Cancer Epidemiol Biomarkers Prev 20: 1918-1924, 2011. 